Research Paper

\title{
Prognostic impact of hormone receptor- and HER2-defined subtypes in inflammatory breast cancer treated with high-dose chemotherapy: a retrospective study
}

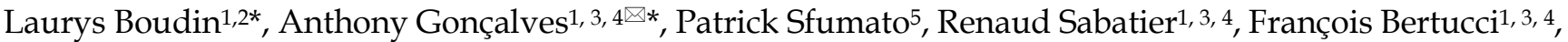 \\ Carole Tarpin ${ }^{1}$, Magali Provansal ${ }^{1}$, Gilles Houvenaeghel ${ }^{3,4}$, 6, Eric Lambaudie $3,4,6$, Agnes Tallet ${ }^{7}$, Michel \\ Resbeut $^{7}$, Emmanuelle Charafe-Jauffret 3 , 4, 8, Boris Calmels $2,9,10$, Claude Lemarie ${ }^{9,10}$, Jean-Marie Boher ${ }^{5}$, \\ Jean-Marc Extra1, Patrice Viens 1, 3,4, Christian Chabannon ${ }^{4,9,10}$ \\ 1. Département d'Oncologie médicale, Institut Paoli-Calmettes (IPC), Marseille, F-13273, France. \\ 2. Département d'Oncologie médicale, Hôpital d'Instruction des Armées Sainte Anne, Toulon, 83000, France. \\ 3. Centre de Recherches en Cancérologie de Marseille (CRCM), UMR Inserm 1068 / CNRS 7258 / AMU 105 / IPC, Marseille, F-13009, France. \\ 4. Aix-Marseille Université, Marseille, F-13284, France. \\ 5. Biostatistiques, Département de la Recherche Clinique et de l'Innovation (DRCI), Institut Paoli-Calmettes, Marseille, F-13273, France. \\ Département de Chirurgie Oncologique, Institut Paoli-Calmettes, Marseille, F -13273, France. \\ Département de Radiothérapie, Institut Paoli-Calmettes, Marseille, F-13273, France. \\ Biopathologie, Département de Biologie du Cancer Institut Paoli-Calmettes, Marseille, F-13273, France. \\ Centre de Thérapie Cellulaire, Département de Biologie du Cancer, Institut Paoli-Calmettes, Marseille, F-13273, France. \\ 10. Centre d'Investigations Cliniques en Biothérapies, Inserm CBT-1409, Marseille, F-13009, France. \\ *both authors equally contribute.
}

$\square$ Corresponding author: Anthony Gonçalves, MD, Ph.D. Department of Medical Oncology. Institut Paoli-Calmettes. 232, boulevard Sainte-Marguerite. 13273 Marseille cedex 9. Phone: +33491223 789. Fax: +33 491223 670. E-mail: goncalvesa@ipc.unicancer.fr

() Ivyspring International Publisher. Reproduction is permitted for personal, noncommercial use, provided that the article is in whole, unmodified, and properly cited. See http://ivyspring.com/terms for terms and conditions.

Received: 2016.04.11; Accepted: 2016.09.05; Published: 2016.10.23

\begin{abstract}
Purpose: Studies examining high-dose chemotherapy with autologous hematopoietic stem cell transplantation (HDC-AHSCT) strategies in inflammatory breast cancer (IBC), showed encouraging results in terms of disease-free survival (DFS), and overall survival (OS). The lack of data regarding HER2 status in all of these studies prevented any prognostic analysis involving breast cancer subtypes.

Methods: All consecutive female patients treated for IBC with HDC and AHSCT at Institut Paoli-Calmettes between 2003 and 2012 were included. Since 2005, trastuzumab was included in initial treatment. Patient, tumor and treatment characteristics were collected. Patients were categorized in three subtypes based on hormonal receptor (HR) and HER2 status of the primary tumor: Luminal, (HR+/HER2-), HER2 (HER2+, any HR), and triple negative (TN) (HER2- and HR-). The main objective was the analysis of OS according to the IHC subtypes.

Results: Sixty-seven patients were included. Eleven patients received trastuzumab. Median follow up was 80.04 months $(95 \% \mathrm{Cl} 73.2-88.08)$. Five-year OS and DFS for the whole population patients were $74 \%(95 \% \mathrm{Cl} 61-83)$ and $65 \%(95 \% \mathrm{Cl} 52-75)$, respectively. OS differed across subtypes $(\mathrm{p}=0.057)$ : HER2 subgroup appeared to have the best prognosis with a 5-year OS of $89 \%(95 \% \mathrm{Cl} 64-97)$ compared to $57 \%(95 \% \mathrm{Cl} 33-76)$ for the TN subgroup (HR 5.38, 95\% Cl 1.14-25.44; $\mathrm{p}=0.034)$.

Conclusions: In IBC patients receiving HDC-AHSCT, OS favorably compares with data available in the literature on similar groups of patients. TN patients carried the least favourable OS and HER2 patients, half of them also receiving trastuzumab, had the best outcome. These findings provide additional information and options for patients with IBC and who could potentially benefit of HDC-AHSCT.
\end{abstract}

Key words: Inflammatory breast cancer; High dose chemotherapy; Immunohistochemical subtypes; Autologous hematopoietic stem cell transplantation. 


\section{Introduction}

Inflammatory breast cancer (IBC), a very aggressive form of locally advanced breast cancer (BC), represent $1 \%$ to $5 \%$ of all $\mathrm{BC}$ cases and differs from other clinical forms both epidemiologically and histolo-clinically [1][2]. Despite dramatic improvement in survival following multimodal treatment including primary chemotherapy (CT) followed by surgery and radiation, IBC prognosis remains poor with 10-year survival ranging from 37 to $55 \%$ [3]. Amongst the many therapeutic options evaluated in IBC, high-dose chemotherapy with autologous hematopoietic stem cells transplantation (HDC-AHSCT) has a special place. Disappointing results of conventional chemotherapy, demonstrated dose response and dose intensity relationships with alkylating agents [4][5] and prognostic impact of initial response to neo adjuvant chemotherapy led to several investigations of HDC strategies in the 90's with encouraging results [6] [7] [8] [9]. The main phase 2 study conducted to completion (PEGASE 02) showed $32 \%$ of pathological complete responses (pCR) post HDC and a 3-year overall survival (OS) rate of $70 \%$ [10]. Despite these promising results, further confirmed by the PEGASE 07 phase 3 study [11], highly significant toxicities leading to premature termination of another phase 2 trial (PEGASE 05 study) prevented the integration of HDC-AHSCT as a standard of care [12]. Thus, in this setting, HDC-AHCST remains experimental and limited to expert centers. However, AHSCT with reinjection of circulating and not bone marrow stem cells, as well as supportive care has achieved a large reduction in procedure-related toxicity and has become substantially less toxic over time [13]. Moreover, in the targeted therapies era, immunohistochemical (IHC) status of IBC is a fundamental data, both for prognosis and treatment. To our knowledge, all studies evaluating HDC-AHSCT for IBC had the common feature of a lack of information about tumor Human Epidermal Growth Factor 2 (HER2) status, which prevented any prognostic analysis involving subtypes. Thus, these findings support the hypothesis that HDC-AHSCT may still have a role in the management of IBC in some selected patients. Hormone receptor and HER 2 status could be potential biomarkers: a meta-analysis of adjuvant studies indicates a possible OS benefit from HDC in patients harboring HER2 negative (HER2-) tumors [14]. The primary objective of this study was to evaluate OS according to IHC-defined molecular subtypes in a recent patient population treated with this strategy. Secondary objectives included disease free survival (DFS) and tolerance of the procedure.

\section{Patients and Methods}

\section{Patient population}

The patient population was identified from our prospectively maintained institutional cell therapy database. Inclusion criteria were as follows: all consecutive female patients treated for IBC with HDC and AHSCT at Institut Paoli-Calmettes between 2003 (the year from which testing for HER2 overexpression was carried out systematically) and 2012. Patient, tumor and treatment characteristics were collected. Minimum criteria required for the diagnosis of IBC included the following: $\mathrm{T} 4 \mathrm{~d}$ according to American Joint Committee on Cancer ( $7^{\text {th }}$ edition) breast cancer staging, characterised by diffuse erythema and oedema, PEV2 (inflammation, erythema, and/or edema localized to $<50 \%$ of the breast surface), or PEV3 (generalized inflammation and edema occupying $>50 \%$ of the breast surface) according to the Institut Gustave-Roussy classification. Metastatic patients were excluded. A flowchart illustrating consecutive steps in the selection process is provided in figure 1.

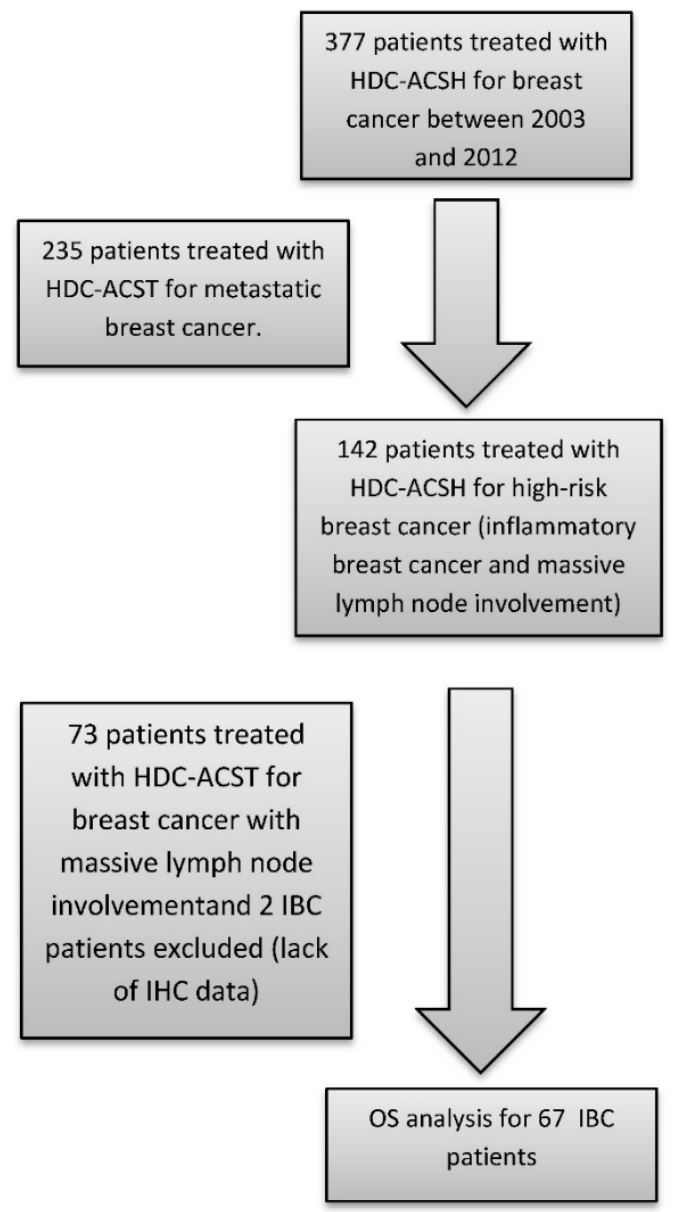

Figure 1: Flow-chart identifying steps for patient selection. HDC: high-dose chemotherapy / ASCT: autologous hematopoietic stem cell transplantation / OS: overall survival IBC: Inflammatory breast cancer / IHC: Immunohistochemical. 
The study was approved by the Institut Paoli-Calmettes (IPC) Institutional Review Board (IRB, Comité d'Orientation Stratégique, COS). All patients undergoing HDC-AHSCT are required to provide signed informed consent, which covers the use and transfer of anonymized data for further clinical research. No further consent was requested by the IRB for the conduct of the present study.

\section{Clinical and biological variables}

Details about patient characteristics, tumor and treatments were recorded. Hormonal receptors (HR) were considered as positive when nuclear staining $\geq 10 \%$ of estrogen receptor (ER) or progesterone receptor (PR) was observed on IHC. HER2 positivity (HER2+) was defined as $3+$ receptor overexpression on IHC staining or $2+$ receptor overexpression on IHC and gene amplification by fluorescence in situ hybridization. Patients were classified into 3 IHC-defined molecular subtypes according to HR and HER2 status: HER2 (HER2+, any HR), luminal (HR+ and HER2-) and triple negative (TN) (HR- and HER2-). Pathological complete response (pCR) was defined as the absence of residual invasive disease in both breast and lymph nodes. Adverse events were assessed using the CTC AE v4.0 criteria.

\section{Treatment procedures}

Patients received HDC-AHSCT in neo adjuvant setting either after conventional therapy or as sequential front line treatment. Conventional chemotherapy comprised four cycles of epirubicin $(100 \mathrm{mg} / \mathrm{m} 2)+$ cyclophosphamide $(500 \mathrm{mg} / \mathrm{m} 2)+5$ fluorouracil $(500 \mathrm{mg} / \mathrm{m} 2)$ followed by 4 cycles of docetaxel $(100 \mathrm{mg} / \mathrm{m} 2)$ associated with trastuzumab ( $8 \mathrm{mg} / \mathrm{kg}$ loading dose then $6 \mathrm{mg} / \mathrm{kg}$ every 21 days) for HER2-positive tumors from since 2005. When it was administered after conventional chemotherapy, HDC was performed after haematological recovery. When administered as sequential front line treatment, HDC comprised 4 cycles of high-dose epirubicine (150 $\left.\mathrm{mg} / \mathrm{m}^{2}\right)+$ cyclophosphamide $\left(4000 \mathrm{mg} / \mathrm{m}^{2}\right)$ each followed by AHSCT and administered after haematological recovery. HDC was followed by surgery, radiotherapy, hormonal therapy in patients with positive hormonal receptors, and adjuvant 1-year trastuzumab for HER2-positive tumors, since 2005. HDC regimens used in this patient population are presented as supplementary material available on line (Supplementary table 1). AHSCT was performed exclusively with peripheral blood cell grafts, as per institutional procedures. As per institutional procedures, CD34+ cells were mobilized using daily administration of rhG-CSF, blood cells were collected using a SPECTRA or OPTIA cell separator, transferred to the cell processing facility onsite, cryopreserved and stored in the vapour phase of liquid nitrogen until distribution. Before administration, cryopreserved cell products were thawed and washed in the cell processing facility, pooled into a single product that was quickly transferred to the clinical ward and administered to the patient the day after HDC. A minimal number of $2 \times 10^{6} \mathrm{CD} 34+$ cells, and whenever possible an optimal number of 5x106 CD34+ cells were collection targets for each autologous transplantation. rhG-CSF was administered only to those women who received less than $2 \times 10^{6}$ CD34+ cells, as measured on the infused pooled, thawed and washed cell product. Since 2005, trastuzumab was included in initial treatment. Endocrine therapy was administered when appropriate as adjuvant treatment either by tamoxifen or aromatase inhibitors according to the menopausal status.

\section{Statistics}

The main objective was the analysis of OS according to the IHC subtypes. Secondary objectives were DFS analysis and toxicity analysis. Descriptive statistics were used to describe the categorical (counts and frequency) and continuous (median and ranges) variables. We tested the difference using a Fisher's exact whenever the condition for chi-square was not reach (expected frequency for each cell $>5$ ) and conserved the chi-square test in other cases. OS was defined as the time from HDC to death as a result of any cause. DFS was defined as the time from HDC to relapse or death as a result of any cause. Patients who were alive at the time of the analysis were censured on the date of last news. Survival curves were generated using Kaplan-Meier method and compared using the Log-rank test. Prognostic impact of the different clinical and biological factors was tested by a Cox regression model in univariate analyses. The level of statistical significance was set at a P-value of 0.05 . Adjusted effect of IHC status on OS according to other prognostic variable(s) which differs between different IHC subgroup ( $p$ value below 0.10 ) was examined by multivariate analysis using Cox's proportional hazard regression model. Statistical analyses were carried out with the $\mathrm{R}$ software version 2.15.2. We followed the "Strengthening the Reporting of Observational Studies in Epidemiology (STROBE)" guidelines [15].

\section{Results}

\section{Patient characteristics}

Sixty-seven patients were included between January 2003 and December 2012. Patient characteristics are summarized in table 1. Patients 
were equally divided in different subgroups with respectively 26, 21 and 20 patients for Luminal, HER2 and TN subgroups. Median age for all patients was 47 (range $24-61$ ) and differed between subgroup with respectively $0,2(9.5 \%)$ and $7(35 \%)$ patients under 35 years for Luminal, HER2 and TN subgroups $(\mathrm{p}<0.01)$. IHC status was available for all patients. Eleven HER2 patients received trastuzumab (52\%).

\section{OS and DFS according to IHC subtypes}

Median follow up was 80.04 months (95\% CI 73.2-88.08). Five-year OS and DFS for the whole population patients were $74 \%$ (95\% CI 61-83) and $65 \%$ (95\% CI 52-75), respectively. The OS curves differed across the different IHC subtypes $(\mathrm{p}=0.057)$ as shown in figure 2. The HER2 subgroup appeared to have the best prognosis with a 5-year OS of $89 \%$ (95\% CI 64-97) compared to $57 \%$ (95\% CI 33-76) for the TN subgroup $(\mathrm{HR}=5.38,95 \%$ CI 1.14-25.44, $\mathrm{p}=0.034)$, whereas the difference was not significant compared with luminal patients $(71 \%, 95 \%$ CI 47-86); $(\mathrm{HR}=2.59,95 \% \mathrm{CI}$ $0.52-12.83, \mathrm{p}=0.244)$. At the time of analysis, all HER 2 patients who received Trastuzumab and 8 of 10 HER2 patients who did not receive trastuzumab were alive. The DFS curves for the different IHC subtypes are shown on figure 3. Five-year DFS for the HER2, luminal and TN subtypes were respectively 74\% (95\% CI $48-88), 60 \%$ (95\% CI 37-77), (HR=1.86, 95\% CI $0.63-5.45, \mathrm{p}=0.260)$ and $58 \%(95 \% \mathrm{CI} 33-76),(\mathrm{HR}=2.12$, 95\% CI 0.69-6.51, $\mathrm{p}=0.187$ ).

In univariate analyses (Table 2), TN subtype was the only parameter significantly associated with OS (HR: 5.38 (95\%CI 1.14-25.44, $\mathrm{p}=0.034)$. For overall survival, the absence of HER2 overexpression was associated with less favorable OS, with borderline significance $(\mathrm{HR}=3.67,95 \% \mathrm{CI} 0.83-16.16, \mathrm{p}=0.066)$. In multivariate analyses (Table 3) including IHC subtype and age (the only prognostic variable which differs between different IHC subgroup), the TN subtype retained an independent poor prognostic value compared to the HER 2 subtype, with a hazard ratio of $6.07(95 \% \mathrm{CI} 1.25-29.4, \mathrm{p}=0.0251)$.

Table 1: Baseline characteristics of patients according to IHC subgroup.

\begin{tabular}{|c|c|c|c|c|c|}
\hline & & LUMINAL (n=26) & HER2 $(n=21)$ & TN $(n=20)$ & p-value* \\
\hline \multirow[t]{3}{*}{ Age } & $<35$ & 0 & $2(9.524)$ & $7(35.00)$ & $<0.001$ \\
\hline & $>=35$ & $26(100.0)$ & $19(90.48)$ & $13(65.00)$ & \\
\hline & Number of missing data & 0 & 0 & 0 & \\
\hline \multirow[t]{3}{*}{ Menopausal status } & Pre & $11(47.83)$ & $10(52.63)$ & $15(75.00)$ & 0.1673 \\
\hline & Post & $12(52.17)$ & $9(47.37)$ & $5(25.00)$ & \\
\hline & Number of missing data & 3 & 2 & 0 & \\
\hline \multirow[t]{4}{*}{ SBR grade } & 1 & $1(4.167)$ & $3(15.00)$ & & 0.4876 \\
\hline & 2 & $8(33.33)$ & $6(30.00)$ & $3(21.43)$ & \\
\hline & 3 & $15(62.50)$ & $11(55.00)$ & $11(78.57)$ & \\
\hline & Number of missing data & 2 & 1 & 6 & \\
\hline \multirow[t]{4}{*}{ HDC regimen } & Endoxan/Melphalan & $15(57.69)$ & $12(57.14)$ & $8(40.00)$ & 0.4886 \\
\hline & NEM & 0 & 0 & $1(5.000)$ & \\
\hline & Epirubicine-Endoxan & $11(42.31)$ & $9(42.86)$ & $11(55.00)$ & \\
\hline & Number of missing data & 0 & 0 & 0 & \\
\hline \multirow[t]{3}{*}{ HDCtiming } & After conventional therapy & $17(65.38)$ & $14(66.67)$ & $10(50.00)$ & 0.4694 \\
\hline & Séquentiel front-line & $9(34.62)$ & $7(33.33)$ & $10(50.00)$ & \\
\hline & Number of missing data & 0 & 0 & 0 & \\
\hline \multirow[t]{3}{*}{ Dermal tumor emboli } & Yes & $14(70.00)$ & $13(72.22)$ & $11(64.71)$ & 0.8854 \\
\hline & No & $6(30.00)$ & $5(27.78)$ & $6(35.29)$ & \\
\hline & Number of missing data & 6 & 3 & 3 & \\
\hline \multirow[t]{3}{*}{ pCR } & Yes & $3(15.79)$ & $9(47.37)$ & $5(27.78)$ & 0.1021 \\
\hline & No & $16(84.21)$ & $10(52.63)$ & $13(72.22)$ & \\
\hline & Number of missing data & 7 & 2 & 2 & \\
\hline \multirow[t]{3}{*}{ Histological subtype } & Ductal & $22(88.00)$ & $18(90.00)$ & $16(88.89)$ & 0.9999 \\
\hline & Other & $3(12.00)$ & $2(10.00)$ & $2(11.11)$ & \\
\hline & Number of missing data & 1 & 1 & 2 & \\
\hline \multirow[t]{4}{*}{ N Stage } & N0 & $8(32.00)$ & $11(55.00)$ & $5(31.25)$ & 0.5109 \\
\hline & N1 & $15(60.00)$ & $8(40.00)$ & $9(56.25)$ & \\
\hline & $\mathrm{N} 2$ & $2(8.000)$ & $1(5.000)$ & $2(12.50)$ & \\
\hline & Number of missing data & 1 & 1 & 4 & \\
\hline
\end{tabular}

IHC: Immuno histo chemical / TN: Triple Negative / HER2: Overexpression of human epidermal growth factor receptor 2 / NEM: Endoxan + Melphalan + Mitoxantrone /HDC: High dose Chemotherapy / pCR Pathological complete response.

${ }^{*}$ Chi-square or Fisher's exact test according to expected scores (see Patient and Methods part). 

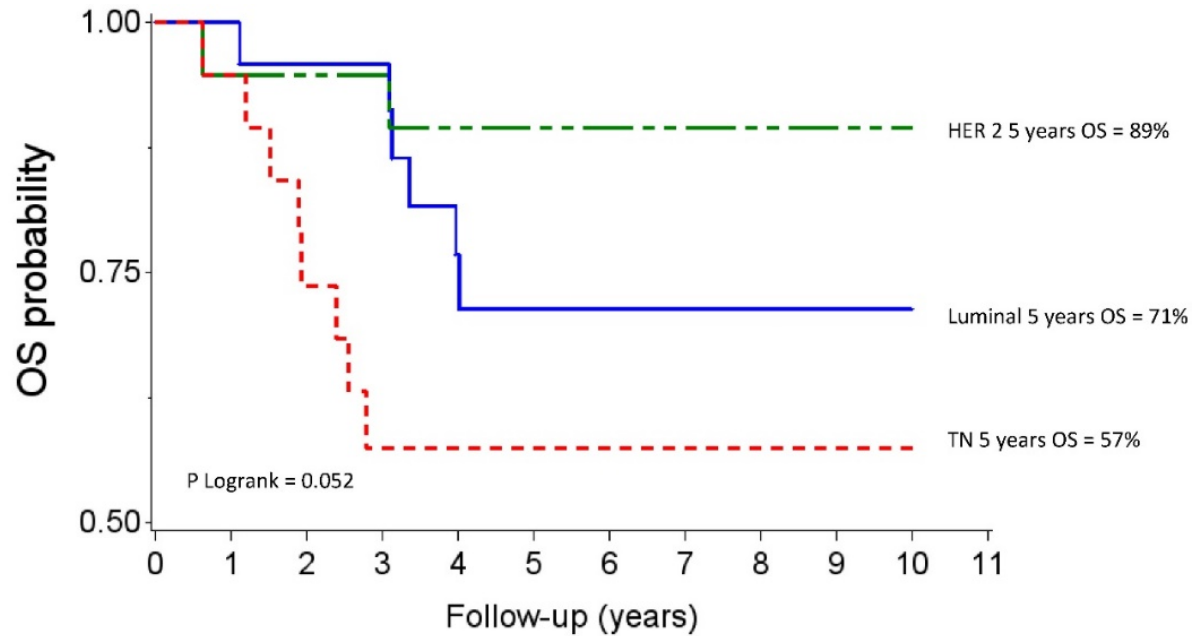

$\begin{array}{llllllllllll}\text { Luminal } & 26 & 24 & 22 & 21 & 16 & 12 & 10 & 6 & 4 & 3 & 1 \\ \text { HER2 } & 21 & 18 & 18 & 18 & 16 & 14 & 12 & 8 & 6 & 4 & 4 \\ \text { TN } & 20 & 18 & 14 & 10 & 10 & 9 & 9 & 6 & 5 & 3 & 2\end{array}$

Figure 2: Overall survival after high-dose chemotherapy and autologous hematopoietic stem cell transplantation in a cohort of 67 inflammatory breast cancer. TN: Triple Negative / HER2: Overexpression of human epidermal growth factor receptor 2.

Table 2: Univariate analysis for prognostic factors for overall survival and disease-free survival after high-dose chemotherapy and autologous hematopoietic stem cell transplantation.

\begin{tabular}{|c|c|c|c|c|c|}
\hline \multirow[t]{2}{*}{ Covariates } & & \multicolumn{2}{|c|}{ OS } & \multicolumn{2}{|c|}{ DFS } \\
\hline & & Hazard ratio & Wald $\mathrm{p}$ value & Hazard ratio & Wald $\mathrm{p}$ value \\
\hline \multirow[t]{2}{*}{ Age } & $<35$ & 1 & & 1 & \\
\hline & $>=35$ & $1.16[0.26,5.12]$ & 0.841 & $1.27[0.38,4.27]$ & 0.699 \\
\hline \multirow[t]{3}{*}{ IHC classification } & HER 2 & 1 & & 1 & \\
\hline & Luminal & $2.59[0.52,12.83]$ & 0.244 & $1.86[0.63,5.45]$ & 0.260 \\
\hline & $\mathrm{TN}$ & $5.38[1.14,25.44]$ & 0.034 & $2.12[0.69,6.51]$ & 0.187 \\
\hline \multirow[t]{2}{*}{ IHC Classification } & HER 2 & 1 & & 1 & \\
\hline & Other & $3.67[0.83,16.16]$ & 0.066 & $1.97[0.73,5.31]$ & 0.182 \\
\hline \multirow{2}{*}{ Dermal tumor emboli } & No & 1 & & 1 & \\
\hline & Yes & $2.48[0.54,11.35]$ & 0.240 & $2.99[0.87,10.21]$ & 0.081 \\
\hline \multirow[t]{2}{*}{ Menopausal status } & Post & 1 & & 1 & \\
\hline & Pre & $1.14[0.41,3.14]$ & 0.800 & $1.04[0.45,2.37]$ & 0.935 \\
\hline \multirow[t]{3}{*}{ HDC regimen } & Endoxan/Melphalan & 1 & & 1 & \\
\hline & NEM & $7.35[0.85,63.43]$ & 0.070 & $3.79[0.48,30.13]$ & 0.208 \\
\hline & Epirubicine-Endoxan & $1.29[0.46,3.63]$ & 0.630 & $1.04[0.45,2.39]$ & 0.934 \\
\hline \multirow[t]{2}{*}{ HDC timing } & After conventional therapy & 1 & & 1 & \\
\hline & Séquentiel front-line & $1.24[0.47,3.31]$ & 0.667 & $1.30[0.57,2.98]$ & 0.528 \\
\hline \multirow[t]{2}{*}{$\mathrm{pCR}$} & Yes & 1 & & 1 & \\
\hline & No & $1.38[0.37,5.09]$ & 0.630 & $1.24[0.44,3.49]$ & 0.681 \\
\hline \multirow[t]{3}{*}{ N Stage } & No & 1 & & 1 & \\
\hline & N1 & $2.42[0.64,9.15]$ & 0.192 & $1.37[0.55,3.43]$ & 0.500 \\
\hline & N2 & $2.39[0.25,22.98]$ & 0.451 & $0.93[0.12,7.48]$ & 0.945 \\
\hline
\end{tabular}

OS: overall survival/ DFS: Disease free survival / IHC: Immuno histo chemical / TN: Triple Negative / HER2: Overexpression of human epidermal growth factor receptor 2 / NEM: Endoxan + Melphalan + Mitoxantrone /HDC: High dose Chemotherapy / ACT Autologous stem cell transplantation/pCR Pathological complete response.

Table 3: Multivariate analysis for prognostic factors for overall survival after high-dose chemotherapy and autologous hematopoietic stem cell transplantation.

\begin{tabular}{llll}
\hline Test & & Hazard ratio & Wald p value \\
\hline IHC subgroup & Luminal vs. HER 2 & $2.46[0.49,12.2]$ & 0.2711 \\
IHC subgroup & TN vs. HER 2 & $6.07[1.25,29.4]$ & 0.0251 \\
Age & $>=35$ vs. $<35$ & $1.78[0.37,8.62]$ & 0.4716 \\
\hline
\end{tabular}

TN: Triple Negative / HER2: Overexpression of human epidermal growth factor receptor 2
Table 4: Extra hematological grade 3 or 4 adverse events during HDC and AHSCT.

\begin{tabular}{ll}
\hline Toxicity & $\mathrm{N}(\%)$ \\
\hline No grade 3 or 4 & $41(59.42)$ \\
Gastro intestinal: & $16(23.18)$ \\
- mucositis & $7(10.14)$ \\
- nausea & $7(10.14)$ \\
- diarrhea & $2(2.89)$ \\
Sepsis & $4(5.79)$ \\
Cardiac & $0(0)$ \\
Pneumopathy & $2(2.89)$ \\
Secondary malignancy & $1(1.45)$ \\
Treatment-related mortality & $0(0)$ \\
\hline HDC and AHSCT: high-dose chemotherapy and autologous hematopoietic stem \\
cell transplantation.
\end{tabular}



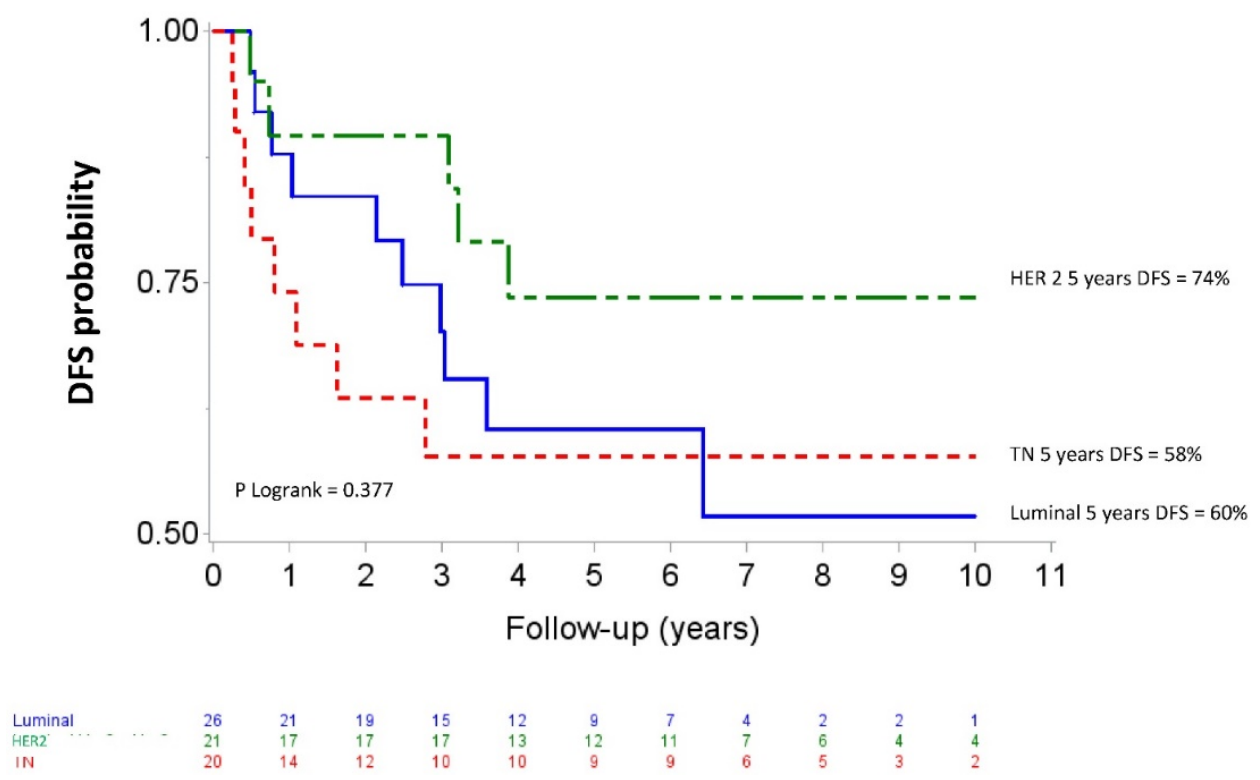

Figure 3: Disease-free survival after high-dose chemotherapy and autologous hematopoietic stem cell transplantation in a cohort of 67 inflammatory breast cancer. TN: Triple Negative / HER2: Overexpression of human epidermal growth factor receptor 2.

\section{Toxicity and post-HDC treatments}

Since HDC is associated with a severe hematological toxicity, which is well described and effectively counterbalanced by AHSCT procedure, we focused on extra-haematological events (Table 4). During the procedure, $40.58 \%$ of patients developed grade 3 or grade 4 non-hematological adverse events, the majority of which being gastrointestinal toxicities $(57.14 \%)$. No patient died from treatment-related events during the HDC-AHSCT. Since a logical concern about HDC-AHSCT is the ability of treated patients to receive subsequent lines of treatment in case of relapse we examined the number of treatment administered in case of progressive disease following HDC-AHSCT. After progression following HDC-AHSCT, a median of 3 lines of treatment (range $0-8)$ was given.

\section{Discussion}

In this retrospective single-center cohort study, we have examined the outcome of a modern population of IBC receiving HDC-AHSCT. With a median follow-up of more than 80 months, 5 -year OS and DFS were 74\% (IC95\% 61-83) and 65\% (IC95\% 52-75), respectively and favorably compared with data available in the literature [16]. TN patients carried the least favourable OS and HER2 patients, half of them also receiving trastuzumab, had the best outcome. Finally, although HDC-AHSCT had a significant extra-haematological toxicity, no treatment-related death occurred and administration of post-progression treatments was not compromised.
To our knowledge, this is the first study to assess the results of HDC-AHSCT in the treatment of IBC for which the IHC-defined breast cancer subtype was known. In this series, DFS and OS results were outstanding for the whole population, but intriguingly, HER2 subgroup had a favorable tendency of survival outcomes (with no significant difference). For these patients, 5- year OS and DFS were $89 \%$ and $74 \%$, respectively, while only $52 \%$ of them received trastuzumab adjuvant treatment. In a recent large retrospective study [17] evaluating 527 IBC patients treated with conventional chemotherapy and for which HER2 and HR status allowed subtyping, 5-year OS ranged from 52.9 to $67.7 \%$ and 5 -year DFS ranged from 26.4 to $35.3 \%$ (depending on HR status) in HER2-positive patients, 55 to $64.5 \%$ of them receiving neoadjuvant and/or adjuvant anti-HER2 treatments. However, regarding to the limited sample size, the more favorable outcome observed in our study should be interpreted with caution.

In the prospective NOAH study, which included HER2-positive locally advanced breast cancer and IBC and randomized the use of neoadjuvant and adjuvant trastuzumab in combination with sequential anthracyclines and taxane-based neoadjuvant chemotherapy, 5-year event-free survival was 58\% (95\% CI 48-66) in patients in the trastuzumab arm [18]. In our study, 5-year- DFS and OS are comparable to those reported in the BEVERLY 2 study at 3 years $(67.8 \%$ and $89.8 \%$ respectively), while all of the BEVERLY 2 study patients received bevacizumab and trastuzumab as neoadjuvant/adjuvant treatment [19]. 
These data suggest possible HDC-AHSCT sensitivity and/or complementarity with trastuzumab in HER2-positive IBC patients.

In our series TN subtype carried the least favorable prognosis, even though the difference did not reach statistical significance which is consistent with previously known data in IBC or non-IBC patient population [17, 20, 21]. However, results were also outstanding for this subtype. Consistently, several data obtained in high-risk early BC suggests an advantage for $\mathrm{HDC}$ in HR-negative patients. A German study showed that BC patients with expression of Y-box-binding protein, which is associated with aggressive tumor phenotype (HR-negative, HER2-positive and p53-postive status, high MIB-1, unfavorable tumor grade) benefited from rapidly cycled tandem high-dose adjuvant chemotherapy [22]. Danebrock-Diallo et al analyzed the predictive and prognostic impact of molecular sub-type in locally advanced BC after HDC dose intensity or conventional regimen. Their conclusion was that the basal and HER2 subgroups benefited from the HDC [23]. Finally, a meta-analysis evaluating randomized trials of HDC in the adjuvant setting of high risk breast cancer (defined by massive lymph node involvement) indicated a possible OS benefit from HDC in patients harboring hormonal or HER2-negative tumors. This benefit appeared greater in the TN population, with an impressive reduction of risk of death by $33 \%$ [24]. In our study 5 -year OS and DFS in the TN subgroup (65\% and 58\%, respectively) also appear significantly higher than those reported in the only study analyzing the outcome of IBC according to their IHC subtype [17] (24.3\% and $17.5 \%$ respectively).

One of the main factors contributing to the near abandonment of the HDC-AHSCT is transplant-related mortality. In this study, no death due to the procedure was noted. Severe non hematologic toxicities were frequent and included mainly mucositis and nausea-vomiting but severe infectious complications also appear limited with $5.79 \%$ of grade 3 or 4 sepsis. In the BEVERLY 2 study, combining conventional chemotherapy to targeted therapies, the rate of grade 3-4 febrile neutropenia was $19 \%$ [19].

Our study has some limitations. This is a single-center and retrospective study, making it difficult to extrapolate its results. Small sample size limits study power to detect significant difference. As there was no control group, which did not undergo HDC-AHSCT, it was impossible to establish whether the favorable outcome of patients was actually due to the HDC-AHSCT itself or to the fact that patient were selected to be "fit" for HDC. Strength of this study is that HR and HER2 status were available for all patients, allowing us to carry out subgroup studies.

\section{Conclusion}

After HDC ACSH for IBC, TN patients carried a tendency for least favourable OS and HER2 patients, the best, with acceptable toxicity. Integration of HDC-AHSCT in the multimodal management of IBC should not be definitively ruled out. In the HER2 subgroup, the potential complementarity of HDC-AHSCT with trastuzumab appears even more promising, with the emergence of HER2 dual inhibition strategies that have shown positive results in BC, including IBC [25].

Finally, a better understanding of the molecular patterns of IBC remains crucial to enable development of personalized treatment strategies in which high-dose alkylating treatments could find a place [26] [27].

\section{Supplementary Material}

Supplementary table 1.

http://www.jcancer.org/v07p2077s1.pdf

\section{Acknowledgement}

This work was supported by SIRIC program (INCa-DGOS-Inserm 6038).

\section{Competing Interests}

The authors have declared that no competing interest exists.

\section{References}

1. Dawood S, Merajver SD, Viens $\mathrm{P}$ et al. International expert panel on inflammatory breast cancer: consensus statement for standardized diagnosis and treatment. Ann. Oncol. Off. J. Eur. Soc. Med. Oncol. ESMO 2011; 22(3):515-523.

2. Monneur A, Bertucci F, Viens P, Gonçalves A. [Systemic treatments of inflammatory breast cancer: an overview]. Bull. Cancer (Paris) 2014; 101(12):1080-1088

3. Rueth NM, Lin HY, Bedrosian I et al. Underuse of trimodality treatment affects survival for patients with inflammatory breast cancer: an analysis of treatment and survival trends from the National Cancer Database. J. Clin. Oncol. Off. J. Am. Soc. Clin. Oncol. 2014; 32(19):2018-2024.

4. Frei E, Teicher BA, Holden SA et al. Preclinical studies and clinical correlation of the effect of alkylating dose. Cancer Res. 1988; 48(22):6417-6423.

5. Frei E, Canellos GP. Dose: a critical factor in cancer chemotherapy. Am. J. Med. 1980; 69(4):585-594.

6. Adkins D, Brown R, Trinkaus $\mathrm{K}$ et al. Outcomes of high-dose chemotherapy and autologous stem-cell transplantation in stage IIIB inflammatory breast cancer. J. Clin. Oncol. Off. J. Am. Soc. Clin. Oncol. 1999; 17(7):2006-2014.

7. Viens P, Penault-Llorca F, Jacquemier J et al. High-dose chemotherapy and haematopoietic stem cell transplantation for inflammatory breast cancer: pathologic response and outcome. Bone Marrow Transplant. 1998; 21(3):249-254.

8. Bertucci F, Tarpin C, Charafe-Jauffret E et al. Multivariate analysis of survival in inflammatory breast cancer: impact of intensity of chemotherapy in multimodality treatment. Bone Marrow Transplant. 2004; 33(9):913-920.

9. Somlo G, Frankel P, Chow W et al. Prognostic indicators and survival in patients with stage IIIB inflammatory breast carcinoma after dose-intense chemotherapy. J. Clin. Oncol. Off. J. Am. Soc. Clin. Oncol. 2004; 22(10):1839-1848.

10. Viens $\mathrm{P}$, Palangié $\mathrm{T}$, Janvier $\mathrm{M}$ et al. First-line high-dose sequential chemotherapy with rG-CSF and repeated blood stem cell transplantation in untreated inflammatory breast cancer: toxicity and response (PEGASE 02 trial). Br. J. Cancer 1999; 81(3):449-456. 
11. Gonçalves A, Pierga J-Y, Ferrero J-M et al. UNICANCER-PEGASE 07 study: a randomized phase III trial evaluating postoperative docetaxel-5FU regimen after neoadjuvant dose-intense chemotherapy for treatment of inflammatory breast cancer. Ann. Oncol. Off. J. Eur. Soc. Med. Oncol. ESMO 2015; 26(8):1692-1697.

12. Palangie $\mathrm{T}$, Viens $\mathrm{P}$, Roche $\mathrm{H}$ et al. Dose-intensified chemotherapy and additional Docetaxel may improve inflammatory breast cancer patients outcome over two decades: Results from Institut Curie protocols 1977-1987 and two consecutive French multicenter trials Pegase 02 (1995-96) and Pegase 05 (1997-99). ASCO Meet. Abstr. 2004; 22(14_suppl):848.

13. Martino M, Bottini A, Rosti G et al. Critical issues on high-dose chemotherapy with autologous hematopoietic progenitor cell transplantation in breast cancer patients. Expert Opin. Biol. Ther. 2012; 12(11):1505-1515.

14. Berry DA, Ueno NT, Johnson MM et al. High-dose chemotherapy with autologous stem-cell support as adjuvant therapy in breast cancer: overview of 15 randomized trials. J. Clin. Oncol. Off. J. Am. Soc. Clin. Oncol. 2011; 29(24):3214-3223.

15. von Elm E, Altman DG, Egger M et al. The Strengthening the Reporting of Observational Studies in Epidemiology (STROBE) Statement: Guidelines for Reporting Observational Studies. PLoS Med. 2007. doi:10.1371/journal.pmed.0040296.

16. Hance KW, Anderson WF, Devesa SS et al. Trends in inflammatory breast carcinoma incidence and survival: the surveillance, epidemiology, and end results program at the National Cancer Institute. J. Natl. Cancer Inst. 2005; 97(13):966-975.

17. Masuda H, Brewer TM, Liu DD et al. Long-term treatment efficacy in primary inflammatory breast cancer by hormonal receptor- and HER2-defined subtypes. Ann. Oncol. Off. J. Eur. Soc. Med. Oncol. ESMO 2014; 25(2):384-391.

18. Gianni L, Eiermann W, Semiglazov V et al. Neoadjuvant chemotherapy with trastuzumab followed by adjuvant trastuzumab versus neoadjuvant chemotherapy alone, in patients with HER2-positive locally advanced breast cancer (the NOAH trial): a randomised controlled superiority trial with a parallel HER2-negative cohort. Lancet Lond. Engl. 2010; 375(9712):377-384.

19. Pierga J-Y, Petit T, Delozier T et al. Neoadjuvant bevacizumab, trastuzumab, and chemotherapy for primary inflammatory HER2-positive breast cancer (BEVERLY-2): an open-label, single-arm phase 2 study. Lancet Oncol. 2012; 13(4):375-384.

20. Kast K, Link T, Friedrich $\mathrm{K}$ et al. Impact of breast cancer subtypes and patterns of metastasis on outcome. Breast Cancer Res. Treat. 2015; 150(3):621-629.

21. Qiu J, Xue X, Hu C et al. Comparison of Clinicopathological Features and Prognosis in Triple-Negative and Non-Triple Negative Breast Cancer. J. Cancer 2016; 7(2):167-173.

22. Gluz O, Mengele K, Schmitt M et al. Y-box-binding protein YB-1 identifies high-risk patients with primary breast cancer benefiting from rapidly cycled tandem high-dose adjuvant chemotherapy. J. Clin. Oncol. Off. J. Am. Soc. Clin. Oncol. 2009; 27(36):6144-6151.

23. Diallo-Danebrock R, Ting E, Gluz O et al. [Prognostic and predictive impact of protein expression profiling in high risk breast cancer patients treated with high-dose chemotherapy]. Verhandlungen Dtsch. Ges. Für Pathol. 2007; 91:187-196.

24. Berry DA, Ueno NT, Johnson MM et al. High-dose chemotherapy with autologous hematopoietic stem-cell transplantation in metastatic breast cancer: overview of six randomized trials. J. Clin. Oncol. Off. J. Am. Soc. Clin. Oncol. 2011; 29(24):3224-3231.

25. Gianni L, Pienkowski T, Im Y-H et al. Efficacy and safety of neoadjuvant pertuzumab and trastuzumab in women with locally advanced, inflammatory, or early HER2-positive breast cancer (NeoSphere): a randomised multicentre, open-label, phase 2 trial. Lancet Oncol. 2012; 13(1):25-32.

26. Bertucci F, Finetti P, Birnbaum D, Viens P. Gene expression profiling of inflammatory breast cancer. Cancer 2010; 116(11 Suppl):2783-2793.

27. Bertucci F, Ueno NT, Finetti P et al. Gene expression profiles of inflammatory breast cancer: correlation with response to neoadjuvant chemotherapy and metastasis-free survival. Ann. Oncol. Off. J. Eur. Soc. Med. Oncol. ESMO 2014; 25(2):358-365 